\title{
The Design and Implementation of Virtual Guide for Taif University based on Semantic Web
}

\author{
Yasser A. Nada \\ Chairman of Department of computer science, \\ Faculty of Computers and Information Technology, \\ Taif University, K S A,
}

\begin{abstract}
Most computer technologies focus on end users and their problem solving activities rather than machine. This denotes an evolutionary trend even for recent technologies which include semantic web and much closer to final users. Hence, building a virtual guide for students or visitors based on semantic web using XML is targeted.

In this paper, premium services for students and visitor in Taif University are introduced with high professionalism level. Furthermore, innovative solutions to improve these services at faculty and university levels are demonstrated. Also, a virtual image in three-dimensional technique is used in the paper. The proposed system provides two main kinds of service. The first is called Open Navigation Service, which provides a user with free tour in university buildings. The second is called Identify Way, which provide a user with start and end visiting points that may be considered university gate or building. In addition, the proposed system combines three important research topics; Semantic Web, Ontology, and XML.
\end{abstract}

\section{Keywords}

Virtual Guide, Semantic Web, Ontology, XML.

\section{INTRODUCTION}

The Semantic Web is a web that is able to describe things in a way that computers can understand. In ontology technology classes and their instances are used to describe domain concept specific resources, data properties and object properties are used to describe characteristics of resources and relationships between them [1]. A challenge for the Semantic Web is enabling information interoperability between related but heterogeneous ontologies [2].

The paper aims to present The Virtual Guide for Students or Visitor in Taif University as an introduction to Student Services for (Declaration, Mapping and Schedule Lectures) and Navigation Service. Student services are where you can find all the information about college that you need whether it's Lectures, tests, instructions, or anything else.

This paper intends to provide information for students of faculty and university. This system specific video guide allows the Taif University to develop presentations aimed at different prospective student constituencies or Visitors.

The Virtual Guide for Students or Visitor in Taif University features an interactive map backed with the Campus guide Multimedia Engine Custom Interactive Map System to allow Virtual Guide University to update and republish any aspect of the map or even change the base map artwork and interactive hotspots when the campus map artwork is updated. Content-managed custom layers allow for highlighting of buildings/locations or the placement of icons.
I have worked to take the viewer on a walking tour around the campus, and created a walking tour at eye level in all parts of the campus, so that the buildings would all be seen from the same perspective as the pedestrian. This meant that the onscreen image would match as closely as possible the reallife view. We then concentrated most of our time and resources on to the creation of a perfect, photo-realistic depiction of the main building, as this is the acknowledged 'hub' of the university geographical layout, recreating every window, doorway and aspect of that building in highly accurate, 3D detail and then sitting it within a 2D map of the campus.

A virtual guide is a simulation of an existing location, usually composed of a sequence of video images. They also may use other multimedia elements such as sound effects, music, narration, and text.

The virtual guide is often used to describe a variety of video and photographic based media. Panorama indicates an unbroken view, since a panorama can be either a series of photographs or panning video footage. However, the phrases virtual guide has mostly been associated with virtual guides created using still cameras.

Virtual Enterprise (VE) is regarded as the most competitive management model of enterprises that faces the resource of the globe. Globalization leads to an efficient new business paradigm of VE, where companies increasingly concentrate on their core competencies and outsource all other functions to their partners on a project basis. a temporary network that exists through networks and aims to share skills, resources, costs and benefits to achieve one or more projects answering to the market opportunities for products and Such virtual guides are made up of a number of shots taken from a single vantage point [3].

Mapping or digital cartography is the process by which a collection of data is compiled and formatted into a virtual image. The primary function of this technology is to produce maps that give accurate representations of a particular area, detailing major road arteries and other points of interest. The technology also allows the calculation of distances from once place to another.

Though digital mapping can be found in a variety of computer applications, such as Google Earth, the main use of these maps is with the Global Positioning System, or GPS satellite network, used in standard automotive navigation systems.

\section{RELATED WORKS}

In [4] the authors presented the characteristics of disaster management is different in different disaster relief information, knowledge, standardization of operating procedures and the feasibility of the rescue program. Knowledge-sharing and case retrieval is to develop case-based 
intelligent decision support system facing the most important issue. Disaster rescue command for decision-making, the use of Web Ontology Language that state the information and knowledge of characteristics of the earthquake disaster rescue, a case-based reasoning and logic to describe the rescue planning business processes.

This paper [5] has proposed two extensions for the current generation of digital libraries. First, authors proposed to use ontology to represent scholarly information in digital libraries, thus making the libraries be able to share and exchange knowledge in the Semantic Web environment. Second, fuzzy theory is employed to process uncertain scholarly information as the forms of fuzzy ontology and fuzzy queries. A general architecture of digital libraries in the Semantic Web environment has been presented and an experimental system has also been developed to verify our ideas and techniques.

In [6] proposed an ontology-based information retrieval model to improve effectiveness of information retrieval. The ontology embedded in the proposal model is a fuzzy taxonomy generated automatically from the documents.

In [1] authors introduces semantic web technology to the design of civil aviation airport emergency management system and presents implemented details through researching on key technology. The semantic data management has been implemented through developing a simulation system and the rapid generation of rescue scheme has also been realized by means of ontology and rules, which provides aid-decision making of airport with efficient technological support.

\section{THE DESIGN OF THE SYSTEM BASED ON SEMANTIC WEB \\ 3.1 Semantic Web}

The Semantic Web is an extension of the World Wide Web with new technologies and standards that enable interpretation and processing of data and useful information for extraction by a computer. The World Wide Web Consortium (W3C) recommends XML, XML Schema, RDF, RDF Schema and Web Ontology Language (OWL) as standards and tools for the implementation of the Semantic Web [7]. Ontologies work as the main component in knowledge representation for the Semantic Web. It is a data model that represents a set of concepts and the relationships between those concepts within a domain. Building an ontology starting from scratch is not an easy task since it makes heavy demands on time in addition to expert knowledge related to the domain.

\subsection{Knowledge Base}

The core of our proposed system is its knowledge base, which encapsulates the human expertise. In practice, the system is only as good as its knowledge base. Hence, Our knowledge contains domain facts and the rules. Our knowledge is represented in XML format.

However, most systems utilize rule-based approaches. The knowledge engineer, working with the expert, must try to define the possible best structure. Other commonly used approaches include decision trees, blackboard systems and object oriented programming.

The semantics of a representation specify how expressions so constructed should be interpreted (i.e. how meaning can be derived from a form). In the proposed system, the knowledge representation methodology uses XML format. Where, two elements of knowledge, facts and model rules are represented using XML format.

\section{XML}

Extensible Markup Language (XML) is a set of rules for encoding documents in machine-readable form. It is defined in the XML 1.0 Specifications produced by the W3C, and several other related specifications, all gratis open standards.

The design goals of XML emphasize simplicity, generality, and usability over the Internet. It is a textual data format with strong support via Unicode for the languages of the world. Although the design of XML focuses on documents, it is widely used for the representation of arbitrary data structures, for example in web services.

Many application programming interfaces (APIs) have been developed that software developers use to process XML data, and several schema systems exist to aid in the definition of XML-based languages.

Currently, web services interact by passing XML data, with data types specified using XML Schema. Simple Object Access Protocol [SOAP] can be used as the communication protocol [8], and the I/O signatures for web services are given by Web Services Description Language [WSDL] [9]. UDDI stands for Universal Description, Discovery and Integration [10] and provides the means to publish and discover web services through a UDDI registry.

A core data representation format for semantic web is Resource Description Framework (RDF). RDF is a framework for representing information about resources in a graph form. It was primarily intended for representing metadata about WWW resources, such as the title, author, and modification date of a Web page, but it can be used for storing any other data. It is based on triples subject-predicate-object that form graph of data. All data in the semantic web use RDF as the primary representation language. The normative syntax for serializing RDF is XML in the RDF/XML form. Formal semantics of RDF is defined as well.

\subsection{Ontologies in Semantic Web}

Ontology is a data model, which can be used to describe a set of concepts and the relationships between those concepts within a domain. Ontology works as the main component in knowledge representation for the Semantic Web. Research groups in both America and Europe developed Ontology modeling languages as The DARPA Agent Markup Language (DAML) and Ontology Inference Layer (OIL.( The W3C Web Ontology Working Group has considered DAML+OIL as the starting point for the introduction of standardized and accepted ontology language for the Semantic Web as Web Ontology Language (OWL) [11]. OWL has three sublanguages: OWL Full, OWL DL and OWL Lite [12]. Existing Semantic Web ontology can be grouped into the following four major categories: metaontology, comprehensive, upper ontology, systematic domain specific ontology, and simple specialized ontology[13]. The Extracted OWL for the Domain Ontology is shown in figure 1.

For querying RDF data as well as RDFS ontology with knowledge bases, a Simple Protocol and RDF Query Language (SPARQL) are available. SPARQL is SQL-like language, but uses RDF triples and resources for both matching part of the query and for returning results of the query. Since RDFS are built on RDF, SPARQL can be used for querying ontology and knowledge bases directly as well. 
- <owl:Class rdf:ID="Programming_Languages"> $<$ rdfs:label>Programming_Languages $</$ rdfs:label $>$ $</$ owl:Class $>$

- <owl:Class rdf:ID="Functions_and_Foundations"> $\langle$ rdfs:label $>$ Functions_and_Foundations $</$ rdfs:label $>$ $<$ rdfs:subClassOf rdf:resource="\#Programming_Languages" | > $</$ owl:Class>

- <owl:Class rdf:ID="Introduction">

$<$ rdfs:label>Introduction</rdfs:label>

$\langle$ rdfs:subClassOf rdf:resource="\#Functions_and_Foundations" / > $</$ owl:Class $>$

- <owl:Class rdf:ID="Programming_Language">

$<$ rdfs:label $>$ Programming_Language $</$ rdfs:label $>$

$<$ rdfs:subClassOf rdf:resource="\#Introduction" />

$</$ owl:Class $>$

- <owl:Class rdf:ID="Goals">

$<$ rdfs:label $>$ Goals $</$ rdfs:label $>$

$<$ rdfs:subClassOf rdf:resource="\#Introduction" />

$</$ owl:Class $>$

- <owl:Class rdf:ID="Programming_Language_History"> <rdfs:label>Programming Language_History $</$ rdfs -label $>$

$<$ rdfs:subClassOf rdf:resource="\#Introduction" / >

$<$ owl:Class >

- <owl:Class rdf:ID="Organization_Concepts_and_Languages"> $<$ rdfs:label>Organization_Concepts and Languages $</$ rdfs:label $>$ $\langle$ rdfs:subClassOf rdf:resource="\#Introduction" / >

$</$ owl:Class $>$

- <owl:Class rdf:ID="Computability">

$<$ rdfs:label>Computability</rdfs:label $>$

$\langle$ rdfs:subClassOf rdf:resource="\#Functions_and_Foundations" / $>$ $<$ owl:Class $>$

- <owl:Class rdf:ID="Partial_Functions_and_Computability"> $<$ rdfs:label $>$ Partial_Functions_and_Computability $</$ rdfs:label $>$ $<$ rdfs:subClassOf rdf:resource="\#Computability" / >

$</$ owl:Class $>$

- <owl:Class rdf:ID="Chapter_Summary">

$<$ rdfs:label>Chapter_Summary</rdfs:label>

$\langle$ rdfs:subClassOf rdf:resource="\#Computability" / $>$

$</$ owl:Class $>$

\section{Figure 1: Extracted OWL for the Domain Ontology}

Note that SPARQL is not only query language; it is also a protocol for accessing RDF data. It is expected that all the semantics and rules will be executed at the layers below Proof and the result will be used to prove deductions. Formal proof together with trusted inputs for the proof will mean that the results can be trusted, which is shown in the top layer of the figure above. For reliable inputs, cryptography means are to be used, such as digital signatures for verification of the origin of the sources. On top of these layers, application with user interface can be built.

\section{SYSTEM ARCHITECTURE 4.1 User Model Acquiring}

A user model is an internal representation of the user's properties. Before a user model can be used it has to be constructed. This process requires many efforts to gather the required information and finally generate a model of the user. The effectiveness of a user profile depends on the information the system delivers to the user. If a large proportion of information is irrelevant, then the system becomes more of an annoyance than a help. This problem can be seen from another point of view; if the system requires a large degree of customization, then the user will not be willing to use it anymore.

The user model saves a system's estimation of what the user has already mastered. This knowledge is then manipulated using inference to get a broader picture of the user's knowledge of the domain. Therefore, we have to redefine the domain and user representations, considering also the uncertainty of knowledge description.

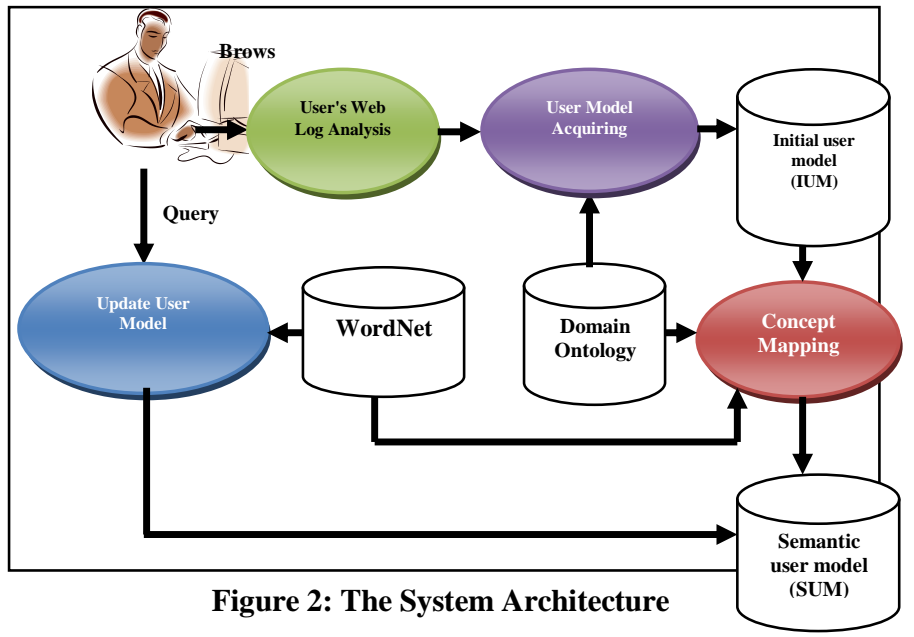

Depending on the content and the amount of information about the user, which is stored in the user profile, a user can be modeled. Thus, the user profile is used to retrieve the needed information to build up a model of the user. The behavior of an adaptive system varies according to the data from the user model and the user profile. Without knowing anything about the user, a system would perform in exactly the same way for all users. Representation of user model [14, $15]$ is a necessary factor for building effective and accurate adaptive systems. Adaptive systems compare user profiles to some reference profiles or item characteristics in order to predict user's model in considering items. The outcome of that process depends on ability to accurately identify and represent user's model.

The presented approach for constructing a semantically enhanced user model that represents the user's interests from web-log data [16] (web usage logs). The goal of incorporating the semantic content of the web pages to build the semantically enhanced user models is to address the high dimensionality problem and semantic inadequacy of the Vector Space Model [17, 18, 19] , on which the initial user model was based, and to map conceptually related terms. To enrich the user model during the user is browsing the pages and navigate web-based system the user model must be updated. To update user model our proposed approach analyzes user's history query keywords by using WordNet.

To acquire user interests we must extract the user behavior and visited page address from web-log data. Then we analyze the visited pages to acquire the terms in the pages that can be considered as concepts in user model. The extracted terms is represented by Vector Space Model that is adapted in our proposed system to achieve effective representations of documents where each document is identified by a ndimensional feature vector where each dimension corresponds to a distinct term. Each term in a given document vector has an associated weight.

The term vector serves as the initial term-based user model (IUM) upon which we intended to improve. To build a semantically enhanced user model (SUM), we used refined domain-specific concepts. First we obtained a list of domainspecific concepts from domain ontology. Then we performed term-to concept mapping between terms in the initial user model (IT-UM) and domain related concepts based on concept hierarchies in WordNet. The final product is a semantically enhanced user model (SUM) in which terms are mapped to related high-level concepts. 
The system architecture is shown in figure 2 .

\subsection{User Ontology Creating}

The user ontology is the specifications of the conceptualization of user information, which defines the various terms relationship related to the user concept, and gives the semantics of the term. User profile ontology mainly describes the interests of the user and display the different attributes and relationships of the user interests. The relation between ontology and semantic web shown in figure3.

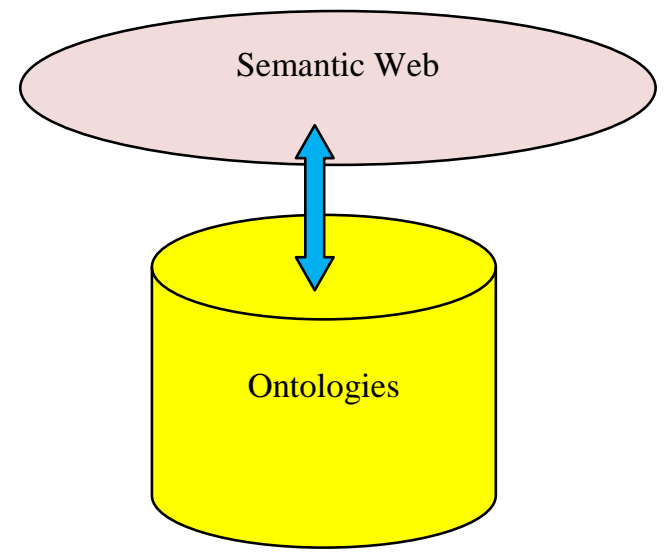

Figure3: Semantic Web and Ontology

In this paper the model we took the domain of software is an XML Semantic Web website, we implement the data knowledge based in the form of XML - RDF file to contain all the collected software which we will include in the website, the format of the RDF file as follows:

We firstly configure Axis in Web.xml as Figure 4.

<servlet-mapping>

<servlet-name>AxisServlet</servlet-name>

$<$ url-pattern>/servlet/AxisServlet</url-pattern>

$</$ servlet-mapping $>$

$<$ servlet-mapping>

$<$ servlet-name $>$ AxisServlet $</$ servlet-name $>$

$<$ url-pattern $>*$.jws $</$ url-pattern $>$

$</$ servlet-mapping $>$

$<$ servlet-mapping>

<servlet-name > AxisServlet $</$ servlet-name $>$

$<$ url-pattern $>$ /services/*</url-pattern $>$

$</$ servlet-mapping $>$

Figure 4: Represented Knowledge in XML.

\section{RESULTS AND TEST THE SYSTEM}

The system was evaluated with different users, including developers, and staff. The system has validated by experts in the domain of service web. Tests of the system were carried out by the developers to make sure the system would work correctly as well as the system is web based system. Figures $(5,6,7,8,9$ and 10) shows the snapshots of the developed system.

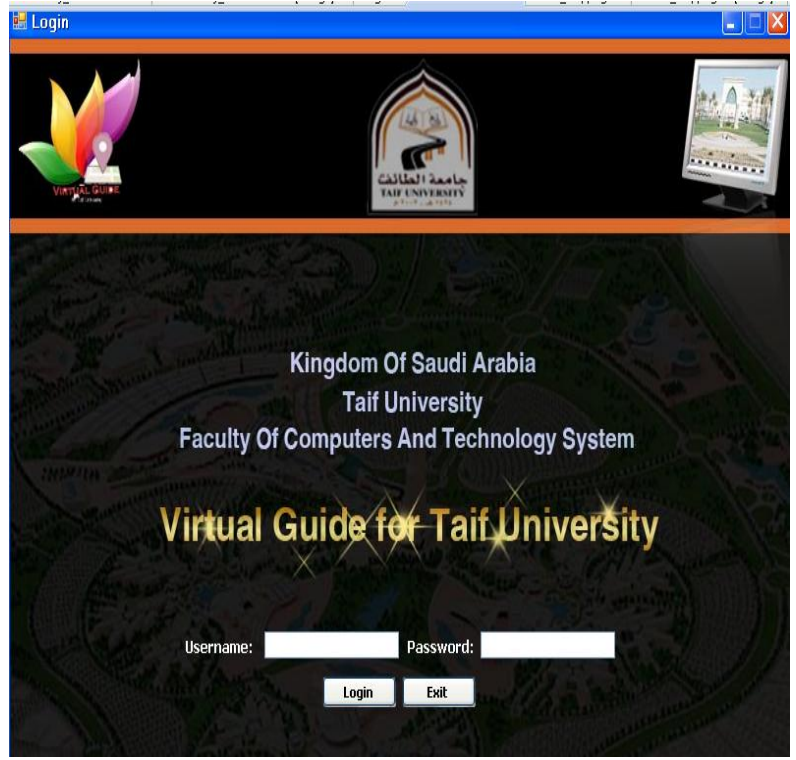

Figure 5: Screen Program Home

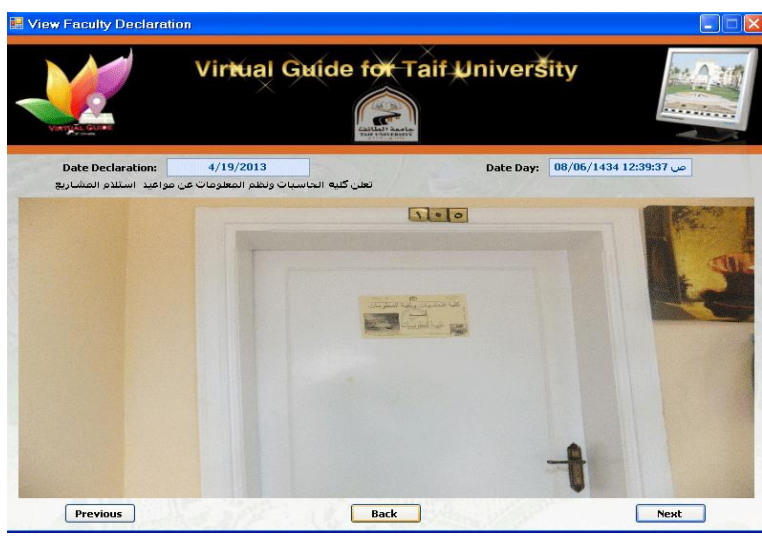

Figure 6: Screen Advertisements College

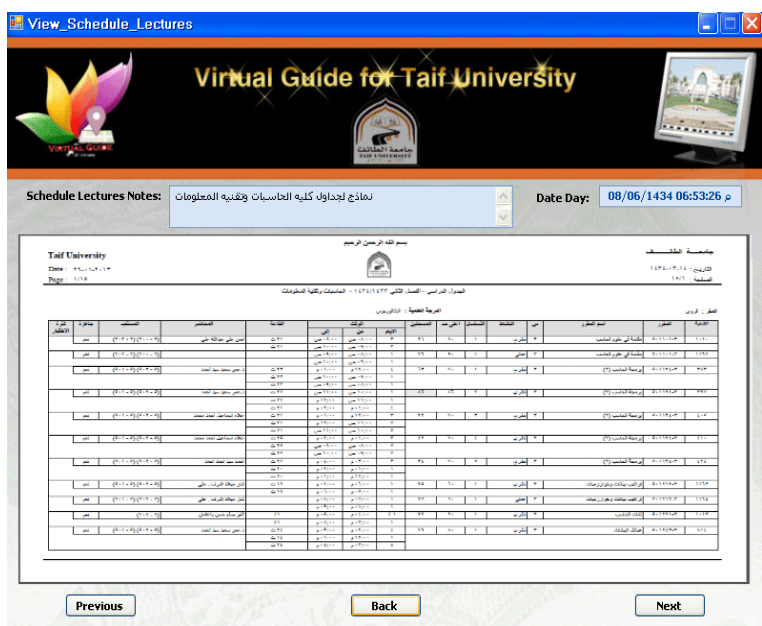

Figure 7: Screen Operating Table 


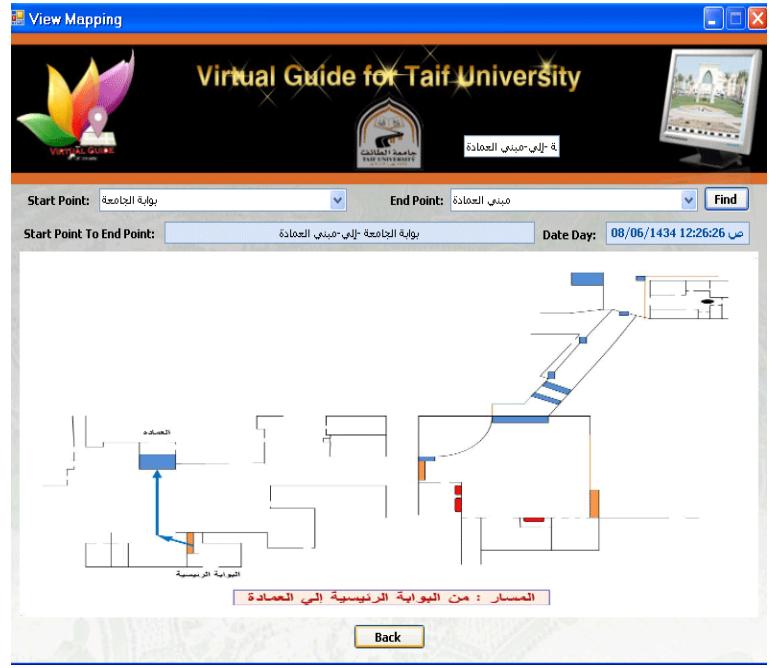

Figure 8: Screen Map

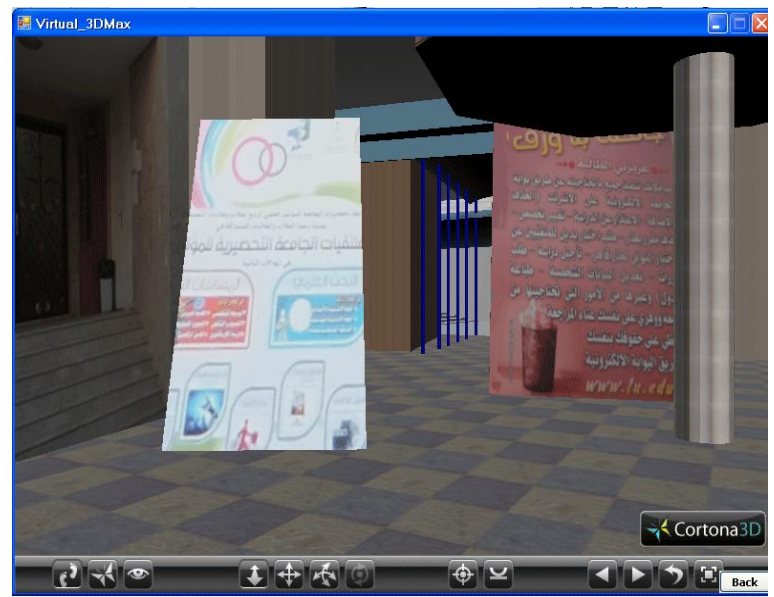

Figure 9: Screen Three Dimensional Start Point

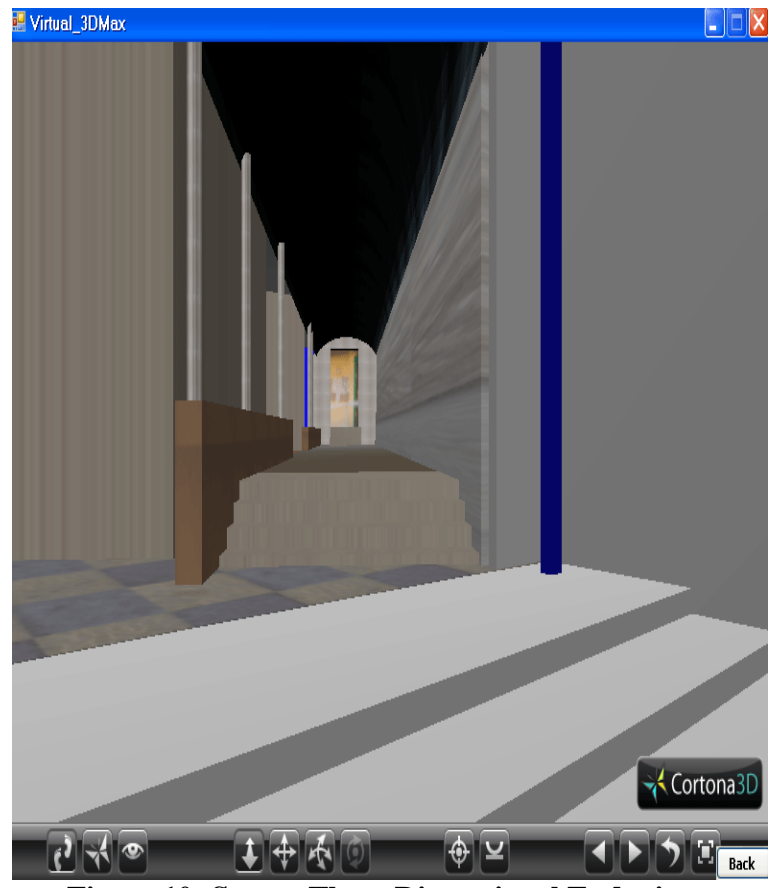

Figure 10: Screen Three Dimensional Endpoint

\section{CONCLUSIONS}

In this paper, Semantic Web is an intelligent web environment in which human and machine agents will communicate semantically. The semantic web has rapidly spread, appearing as a new challenge for knowledge sharing and automatic processing.

The Virtual Guide for Students or Visitor in Taif University in the virtual image using three dimensional techniques. The system provides two main services:

- Open Navigation Service: through that service the user or visitor can tour free to view information about any building.

- Identify ways: via this service the user can choose a starting point from a list. This starting point may be the university gate or any building, and then the user can choose an ending point.

This system contains a part for presentations of the declarations of the university, and the student services can find all the information about college that's needed whether they are Lectures, tests, instructions, or anything else. The Web service provides various advantages for facilitating cooperation between the visitor and university.

So resources are organized in a semantic way which makes computers able to process data automatically and provide information service more intelligently.

\section{REFERENCES}

[1] Hong Wang, Zhenjie Pan, Wentao Zhou., "The Design and Implementation of Information System in Civil Airport Emergency Management Based on Semantic Web", 25th Chinese Control and Decision Conference (CCDC), IEEE 2013.

[2] Valerie Cross, Xueheng Hu. "Fuzzy Set and Semantic Similarity in Ontology Alignment", WCCI 2012 IEEE World Congress on Computational Intelligence, Brisbane, Australia June, 10-15, 2012.

[3] Chouyin Hsu, "Semantic Case-based Reasoning for Virtual Enterprises in Project Management", The IET International Conference on Frontier Computing Theory, Technologies and Applications, 2010.

[4] Wu Yun, Li Chan, "Semantic Web-based Seismic Disaster Management Expert System", Paper 70601011 supported by National Natural Science Foundation of China, 2009.

[5] Q. Tho, H. Siu, and C. Tru , "Ontology-Based Fuzzy Retrieval for Digital Library", ICADL 2007, LNCS 4822 pp. 95-98. Springer-Verlag Berlin Heidelberg, 2007.

[6] C. Been-chian, H. Chih-hung, J. Ming-yi, "Intelligent Information Retrieval Applying Automatic Constructed Fuzzy Ontology", Proceedings of the Sixth International Conference on Machine Learning and Cybernetics, Hong Kong, 19-22 August 2007. 1-4244-0973-X/07, IEEE, 2007.

[7] Berners-Lee T, Hendler J, Lassila O., "The Semantic Web", Scientific American, 284(5): 34 43, 2001.

[8] http://www.w3.org/TR/soap/

[9] http://www.w3.org/TR/wsdl.html

[10] http://www.uddi.org/ 
[11] G. Antoniou, F. V. Harmelen, "Handbook on Ontologies in Information Systems", Springer-Verlag , pp. 76-92, 2003.

[12] L. Deborah McGuinness, F. V. Harmelen, "OWL Web Ontology Language Overview”, W3C Recommendation , Tech. Rep., 10, Feb, 2004.

[13] Li Ding, et al., "Using Ontologies in the Semantic Web: A Survey", Department of Computer Science and Electrical Engineering , University of Maryland Baltimore County, Baltimore, Tech. Rep. TR CS-05-07, July, 2005.

[14] Z. Ning, L. Yuefeng, "Ontology-Based Web Mining Model: Representation of User Profiles", Proceeding of the IEEE/WIC International Conference on Web Intelligence (WI'03), 0-7695-1932-6/03, IEEE ,2003.

[15] S. Ahu, M. Bamshad, B. Robin, "Ontological User Profiles for Representing Context in Web Search", 2007 IEEE/WIC/ACM International Conferences on Web Intelligence and Intelligent Agent Technology Workshops. 0-7695-3028-1, IEEE, 2007.

[16] http://www.w3.org/TR/WD-logfile.html.

[17] F. Christoph, "User Modeling and User Profiling in Adaptive E-learning Systems", Master's Thesis at Graz University of Technology, Copyright by Christoph, 2005.
[18] L. Xiaojian, C. Shihong, "Personalized Query Expansion Based on Semantic User Model in e-Learning System" , 2009 Sixth International Conference on Fuzzy Systems and Knowledge Discovery, DOI 10.1109/FSKD.2009.787, 978-0-7695-3735-1/09, IEEE 2009.

[19] A. Palakorn, H. Hyoil, N. Olfa, J. Roberta, "Semantically Enhanced User Modeling", SAC'07, March 11-15, 2007, Seoul, Korea., ACM 1-59593-480-4/07/0003 , 2007.

\section{AUTHORS BIOGRAPHY}

DR. Yasser Ahmed Nada Was born in Ismailia, Egypt, in 1968. He received the BSc degree in pure Mathematics and Computer Sciences in 1989 and MSc degree for his work in computer science in 2003, all from the Faculty of Science, Suez Canal University, Egypt. In 2007, he received his Ph.D. in Computer Science from the Faculty of Science, Suez Canal University, Egypt. From September 2007 until now, he worked as Associate Professor of computer science. Chair, Department of computer science, Faculty of Computers and Information Technology, Taif University, KSA. His research interests include Expert Systems, Artificial Intelligence, Semantic Web, Object Oriented Programming, Computer Vision, and Genetic. 